\title{
Spectral Functions, Special Functions and the Selberg Zeta Function
}

\author{
A. Voros $\star$ \\ Service de Physique Théorique, CEN-Saclay, F-91191 Gif-sur-Yvette Cedex, France
}

\begin{abstract}
The functional determinant of an eigenvalue sequence, as defined by zeta regularization, can be simply evaluated by quadratures. We apply this procedure to the Selberg trace formula for a compact Riemann surface to find a factorization of the Selberg zeta function into two functional determinants, respectively related to the Laplacian on the compact surface itself, and on the sphere. We also apply our formalism to various explicit eigenvalue sequences, reproducing in a simpler way classical results about the gamma function and the Barnes $G$-function. Concerning the latter, our method explains its connection to the Selberg zeta function and evaluates the related Glaisher-Kinkelin constant $A$.
\end{abstract}

\section{Introduction}

This note studies from a general and systematic point of view certain spectral functions, especially determinants and generalized zeta functions, which can be associated with a numerical sequence $\left\{\lambda_{k}\right\}$ (typically the spectrum of a certain differential operator). In particular we reduce to elementary manipulations the evaluation of certain functional determinants which find applications in high energy physics (string theory, see [1] and references therein) and in differential geometry (analytic torsion, see [2,3] and references therein). Our main result (Sect. 7) is actually an explicit factorization of the Selberg zeta function into two functional determinants, one of which is expressible in terms of the Barnes $G$-function; some recently published formulae involving the Selberg zeta function $[1,3]$ follow from ours by specializing the value of the spectral variable.

Our formalism itself is developed in Sect. 2 to 4; its connection to asymptotic (semi-classical) expansions is demonstrated in Sect. 5, and Sect. 6 is devoted to examples drawn from number theory (the Euler gamma function and the Barnes $G$-function) and from quantum mechanics (homogeneous Schrödinger operators like the quartic oscillator).

\footnotetext{
* Member of CNRS
} 
While the underlying ideas are certainly not new, they are very scattered in the literature, hence we hope that this condensed and systematic presentation (in which, however, we make no pretence of rigour) can give new, simpler viewpoints on the subject. The references given are those which were of direct use to us, and they are not supposed to reflect accurately all original contributions.

\section{Sequences and Associated Functions}

The present work studies several functions which can be conveniently associated with suitable numerical sequences $\left\{\lambda_{k}\right\}$. The eigenvalues of certain interesting operators are the most natural and typical candidates to form admissible sequences $\left\{\lambda_{k}\right\}$; the associated functions are then spectral functions (functions of the spectrum). However, the general theory of those functions only relies on a limited set of properties to be satisfied by the sequence $\left\{\lambda_{k}\right\}$; it is therefore convenient to view the eigenvalue sequence as an abstract sequence of numbers $\left\{\lambda_{k}\right\}$ restricted by a basic set of requirements, which are the following:

$$
0<\lambda_{0} \leqq \lambda_{1} \leqq \lambda_{2} \leqq \cdots ; \quad \lambda_{k} \uparrow+\infty
$$

(strict positivity is crucial at some stages, but zero modes can easily be "removed by hand" when necessary; eigenvalues are to be repeated according to their multiplicities);

2) the following series converges for all $\operatorname{Re} t>0$, defining the partition function,

$$
\Theta(t)=\sum_{k=0}^{\infty} e^{-t \lambda_{k}}
$$

another basic assumption is that $\Theta(t)$ admits for $t \rightarrow 0$ a full asymptotic expansion (i.e. to all orders in $t$ ),

$$
\Theta(t) \sim \sum_{n=0}^{\infty} c_{i_{n}} t^{i_{n}}
$$

for a suitable increasing sequence of (real) exponents $\left\{i_{n}\right\}$, with $\left\{i_{n}\right\} \uparrow+\infty$ and $i_{0}<0$. The coefficients $c_{i_{n}}$ are taken here as known quantities; $c_{i_{0}}$ is positive by necessity. It will prove useful to define, by convention,

$$
c_{\alpha} \equiv 0 \text { for any real number } \alpha \text { not in the sequence }\left\{i_{n}\right\} \text {. }
$$

The divergent leading term $c_{i_{0}} t^{i_{0}}$ in Eq. (1.3) implies that the sequence $\left\{\lambda_{k}\right\}$ satisfies the Weyl estimate

$$
\mathscr{N}(\lambda) \sim \frac{c_{i_{0}}}{\Gamma\left(1-i_{0}\right)} \lambda^{-i_{0}}
$$

where $\mathcal{N}(\lambda)=$ (number of $\lambda_{k} \leqq \lambda$ ) is the counting function, or cumulative distribution, of the sequence. (This follows from Karamata's Tauberian theorem [4] applied to the formula $\left.\Theta(t)=\int_{0}^{\infty} e^{-t \lambda} d \mathscr{N}(\lambda)\right)$. We now introduce a special name for the positive exponent $\left(-i_{0}\right)$ occurring in the estimate (1.5); we call it the order of the sequence, $\mu=-i_{0}$. 
In the present work we shall not be concerned with rigorous proofs, which certainly imply additional regularity properties for the sequence $\left\{\lambda_{k}\right\}$.

This level of generality, where the sequence $\left\{\lambda_{k}\right\}$ is only specified by a few abstract properties, will allow for a great variety of concrete applications, encompassing many fields of interest. The spectral functions of several simple numerical sequences reproduce classical functions of analysis. As the next example of interest, the eigenvalues of an elliptic (pseudo-) differential operator of order $m$ on a $d$-dimensional compact (or bounded) manifold form an admissible sequence, of order $\mu=d / m$ [5]. The spectrum of the Schrödinger operator with a positive polynomial potential is another example [6].

In this work we shall mainly be concerned with the following two types of spectral functions and their mutual relationships:

the Fredholm determinant,

$$
\Delta(\lambda)=\prod_{0}^{\infty}\left(1-\lambda / \lambda_{k}\right)
$$

and the zeta function,

$$
\begin{aligned}
Z(s) & =\sum_{0}^{\infty} \lambda_{k}^{-s} \\
& =\frac{1}{\Gamma(s)} \int_{0}^{\infty} \Theta(t) t^{s-1} d t
\end{aligned}
$$

These formal definitions are too restricted in scope, however. The infinite product (1.6) only converges for sequences with $\mu<1$; while both definitions of the zeta function make no restriction on $\mu$, they require that $\operatorname{Re} s>\mu$. (In this region the equivalence of formulae (1.7) and (1.8) follows from the definition (1.2) of $\Theta(t)$.)

For the simpler case $\mu<1$ we refer to our previous works $[6,7]$, while here we emphasize the treatment of an arbitrary order $\mu$. Therefore, two technical digressions are in order at this stage, one on Weierstrass canonical products, and the other on the analytical continuation of Mellin transforms; both consists of standard material and are only presented for completeness.

\section{Regularization Techniques for Infinite Products and Mellin Transforms}

a) Canonical Regularization of Infinite Products [8]. The infinite product

$$
\Delta(\lambda)=\prod\left(1-\lambda / \lambda_{k}\right)
$$

has the same convergence properties as the infinite series

$$
\mathrm{R}(\lambda)=-\frac{d}{d \lambda} \log \Delta(\lambda)=\sum\left(\lambda_{k}-\lambda\right)^{-1}
$$

(this is the trace of the resolvent (i.e. of Green's function) if $\left\{\lambda_{k}\right\}$ are eigenvalues). Convergence is achieved if $\mu<1$ and $\Delta(\lambda)$ is then an entire function of order $\mu$, being specified by its Weierstrass canonical product over the zeros and by the normalization condition $\Delta(0)=1$. 
When $\mu>1$ the analogous Weierstrass product is given by

$$
\Delta(\lambda)=\prod_{k=0}^{\infty}\left(1-\lambda / \lambda_{k}\right) \exp \left(\frac{\lambda}{\lambda_{k}}+\frac{\lambda^{2}}{2 \lambda_{k}^{2}}+\cdots+\frac{\lambda^{[\mu]}}{[\mu] \lambda_{k}^{[\mu]}}\right),
$$

where $[\mu]$ denotes the integer part of $\mu$. This is the unique entire function of order $\mu$ having the zeros $\left\{\lambda_{k}\right\}$ and subjected to the normalization conditions

$$
\log \Delta(0)=\frac{d}{d \lambda} \log \Delta(0)=\cdots=\frac{d^{[\mu]}}{d \lambda^{[\mu]}} \log \Delta(0)=0 .
$$

This is equivalent to defining at first the $[\mu]^{- \text {th }}$ derivative of $\mathrm{R}(\lambda)$ as

$$
\mathrm{R}^{([\mu])}(\lambda)=\mathrm{R}_{[\mu]}(\lambda)=[\mu] ! \sum\left(\lambda_{k}-\lambda\right)^{-[\mu]-1},
$$

which converges, and then defining backwards

$$
\mathrm{R}_{j-1}(\lambda)=\int_{0}^{\lambda} \mathrm{R}_{j}\left(\lambda^{\prime}\right) d \lambda^{\prime}, \ldots, \log \Delta(\lambda)=-\int_{0}^{\lambda} \mathrm{R}_{0}\left(\lambda^{\prime}\right) d \lambda^{\prime} .
$$

The choice of $\lambda=0$ as the lower integration bound at every stage amounts to the normalization conditions (2.4). The choice of a different normalization point $\lambda=-a$ (with $a \geqq-\lambda_{0}$ to avoid ambiguities) can be reduced to a global shift of the sequence, $\left\{\lambda_{k}\right\} \rightarrow\left\{\lambda_{k}+a\right\}$. Setting indeed

$$
\Delta_{a}(\lambda)=\prod_{k=0}^{\infty}\left(1-\frac{\lambda}{\lambda_{k}+a}\right) \exp \left(\frac{\lambda}{\lambda_{k}+a}+\cdots+\frac{\lambda^{[\mu]}}{[\mu]\left(\lambda_{k}+a\right)^{[\mu]}}\right),
$$

we have

$$
\frac{d^{[\mu]+1}}{d \lambda^{[\mu]+1}}\left(-\log \Delta_{a}(\lambda)\right) \equiv \mathrm{R}^{([\mu])}(\lambda-a),
$$

and by integrating backwards we get

$$
-\log \Delta_{a}(\lambda)=-\log \Delta(\lambda-a)-\sum_{m=0}^{[\mu]} \frac{d^{m}}{d \lambda^{m}}[-\log \Delta(\lambda-a)]_{\lambda=0} \lambda^{m} / m !
$$

or, using (2.6),

$$
\Delta_{a}(\lambda)=e^{\sum_{m=1}^{[\mu]} \mathrm{R}_{m}(-a) \lambda^{m} / m !} \Delta(\lambda-a) / \Delta(-a) .
$$

This formula can also be viewed as expressing the Hadamard factorization property for entire functions of order $\mu$ [8].

b) Mellin Transforms [9,10]. If a function $\Phi(t)$ defined on $0<t<\infty$ satisfies the estimates

$$
\begin{aligned}
& \Phi(t)=0\left(t^{i_{0}}\right) \quad \text { for } \quad t \rightarrow+0 \quad\left(i_{0} \leqq+\infty\right), \\
& \Phi(t)=0\left(t^{j_{0}}\right) \text { for } t \rightarrow+\infty \quad\left(-\infty \leqq j_{0}\right) \text {, }
\end{aligned}
$$

and if

$$
j_{0}<i_{0}
$$


then $\Phi(t)$ admits a Mellin transform $\mathscr{M} \Phi(s)$ in the vertical strip $-i_{0}<\operatorname{Re} s<-j_{0}$,

$$
\mathscr{M} \Phi(s)=\int_{0}^{\infty} \Phi(t) t^{s-1} d t
$$

A standard problem is how to continue $\mathscr{M} \Phi(s)$ analytically to a wider strip. Since

$$
-\mathscr{M} \Phi(-s)=\int_{0}^{\infty} \Phi(1 / t) t^{s-1} d r
$$

this problem has a left-right symmetry in $s$, hence we may restrict the problem to the analytical continuation of $\mathscr{M} \Phi(s)$ towards the left, say. If $-i_{0}=-\infty$ the problem is solved, otherwise the obstruction to a straightforward continuation of Eq. (2.12) to $\operatorname{Re} s \leqq i_{0}$ is the singularity of the integrand at $t=0$. We may resolve this singularity if we assume a more precise estimate than (2.9), namely

$$
\Phi(t)=c_{i_{0}} t^{i_{0}}+0\left(t^{i_{1}}\right) \text { for some } i_{1}>i_{0}, \quad t \rightarrow+0 .
$$

We may then integrate $(2.12)$ by parts to obtain

$$
\mathscr{M} \Phi(s)=-\int_{0}^{\infty} \frac{d}{d t}\left(t^{-i_{0}} \Phi(t)\right) \frac{t^{s+i_{0}}}{s+i_{0}} d t
$$

This new expression converges in a wider strip, $-i_{1}<\operatorname{Re} s<-j_{0}$, where it defines a meromorphic function with one simple pole, $s=-i_{0}$, and residue $c_{i_{0}}$.

If now $\Phi(t)$ admits a full asymptotic expansion as $t \rightarrow+0$,

$$
\Phi(t) \sim \sum_{0}^{\infty} c_{i_{n}} t^{i_{n}}, \quad\left\{i_{n}\right\} \uparrow+\infty
$$

then repeated integration by parts shows that $\mathscr{M} \Phi(s)$ has a meromorphic continuation to the whole half-plane $\operatorname{Re} s<j_{0}$, with a sequence of simple poles

$$
s=-i_{n}(\downarrow-\infty), \quad \text { with: Residue }=c_{i_{n}} .
$$

[By the symmetry relation (2.13), if

$$
\Phi(t) \sim \sum_{0}^{\infty} b_{j_{n}} t^{j_{n}} \quad(t \rightarrow+\infty), \quad\left\{j_{n}\right\} \downarrow-\infty,
$$

then $\mathscr{M} \Phi(s)$ extends likewise to $\operatorname{Re} s \geqq-j_{0}$ with the sequence of simple poles

$$
\left.s=-j_{n}(\uparrow+\infty), \quad \text { with: Residue }=-b_{j_{n}} \cdot\right]
$$

\section{Application to the Zeta Functions}

The ordinary zeta function of the sequence $\left\{\lambda_{k}\right\}$ is, by Eq. (1.8),

$$
\mathrm{Z}(s)=\Gamma(s)^{-1} \mathscr{M} \Theta(s),
$$

where $\Theta(t)=\sum_{0}^{\infty} e^{-t \lambda_{k}}$ is the partition function (1.2). Since $\lambda_{0}>0, \Theta(t)$ decreases 
exponentially for $t \rightarrow+\infty$, while it has precisely an expansion like (2.16) for $t \rightarrow+0$, by our basic assumption (1.3). It then follows that $Z(s)=\Gamma(s)^{-1} \mathscr{M} \Theta(s)$ is analytic for $\operatorname{Re} s>-i_{0}(=\mu)$, and meromorphic in the whole $s$ plane with the poles

$$
s=-i_{n}, \quad \text { Residue }=c_{i_{n}} / \Gamma\left(-i_{n}\right) .
$$

The negative or zero integers $s=-m$ are always regular values of $Z(s)$, because $1 / \Gamma(s)$ vanishes there, and

$$
\mathrm{Z}(-m)=(-1)^{m} m ! c_{-m} .
$$

These closed expressions form the trace identities; the convention (1.4) for $c_{-m}$ applies here [11].

It is however more convenient to introduce a two parameter spectral function, or generalized zeta function,

$$
\begin{aligned}
\mathrm{Z}(s, a) & =\sum_{0}^{\infty}\left(\lambda_{k}+a\right)^{-s} \\
& =\Gamma(s)^{-1} \int_{0}^{\infty} \Theta(t) e^{-a t} t^{s-1} d t .
\end{aligned}
$$

Strictly speaking, these formulae make sense for $\operatorname{Re} s>\mu$ and $a \geqq-\lambda_{0}$, and should be analytically continued elsewhere.

It is convenient to use the auxiliary functions

$$
\eta(s, a)=\mathscr{M} \Phi_{a}(s), \quad \Phi_{a}(t)=\Theta(t) e^{-a t},
$$

so that

$$
\mathrm{Z}(s, a)=\Gamma(s)^{-1} \eta(s, a) .
$$

This single function $Z(s, a)$ has the virtue of generating many other useful spectral functions,

$$
\begin{aligned}
& \text { - the ordinary zeta function } \mathrm{Z}(s)=\mathrm{Z}(s, 0) ; \\
& \text { - the trace of the resolvent } \mathrm{R}(\lambda)=\mathrm{Z}(1,-\lambda),
\end{aligned}
$$

(with $-\infty<\lambda<\lambda_{0}$ ), or rather its $n$-th derivative

$$
\frac{d^{n} \mathrm{R}(\lambda)}{d \lambda^{n}}=n ! \mathrm{Z}(n+1,-\lambda)=\eta(n+1,-\lambda),
$$

which makes sense for $n \geqq[\mu]$, where Eq. (3.4) actually converges.

Our next purpose is to give an explicit formula by which $\mathrm{Z}(s, a)$ will also generate the determinant function $\Delta(\lambda)$ of Eq. (2.3). This will make use of the following properties of $\mathrm{Z}(s, a)$ and $\eta(s, a)$.

For fixed $a, \mathrm{Z}(s, a)$ and $\eta(s, a)$ are meromorphic functions in the complex $s$ plane, by the Mellin transform theory applied to $\Phi_{a}(t)=\Theta(t) e^{-a t}$. Their poles and residues are encoded in the expansion of $\Phi_{a}(t)$ for $t \rightarrow+0$ as explained in Eq. (2.17),

$$
\Phi_{a}(t) \sim\left(\sum_{n=0}^{\infty} c_{i_{n}} t^{i_{n}}\right)\left(\sum_{m=0}^{\infty} \frac{(-a)^{m}}{m !} t^{m}\right) .
$$

The trace identities state that $s=-n$ are regular points for $\mathrm{Z}(s, a)$, and that 
$(-1)^{n} \mathrm{Z}(-n, a) / n$ ! is the coefficient of $t^{n}$ in the expansion (3.11); in particular,

$$
\mathrm{Z}(0, a)=\sum_{m=0}^{[\mu]} \frac{c_{-m}}{m !}(-a)^{m}
$$

is a polynomial of degree $[\mu]$ with respect to $a$.

Concerning now the variable $a$, we have an obvious functional relation

$$
\frac{\partial}{\partial a} \mathrm{Z}(s, a)=-s \mathrm{Z}(s+1, a) \Leftrightarrow \frac{\partial}{\partial a} \eta(s, a)=-\eta(s+1, a),
$$

and an asymptotic expansion for $a \rightarrow+\infty$, which is obtained (when $\operatorname{Re} s>\mu$ ) by substituting the small $t$ expansion $\Theta(t) \sim \sum c_{i_{n}}{ }^{i_{n}}$ into Eq. (3.5) and integrating term by term:

$$
\begin{gathered}
\eta(s, a) \sim \sum c_{i_{n}} \int_{0}^{\infty} t^{i_{n}+s-1} e^{-a t} d t, \\
\eta(s, a) \sim \sum_{n=0}^{\infty} c_{i_{n}} \Gamma\left(s+i_{n}\right) a^{-s-i_{n}} \quad\left(\operatorname{Re} s>i_{0}\right) . \\
\mathrm{Z}(s, a) \sim \Gamma(s)^{-1} a^{-s} \sum_{n=0}^{\infty} c_{i_{n}} \Gamma\left(s+i_{n}\right) a^{-i_{n}} \quad\left(\operatorname{Re} s>i_{0}\right) .
\end{gathered}
$$

A crucial fact for our purposes is that this same expansion formula actually extends to the whole s plane. Indeed, if we had substituted $\Theta(t) \sim \sum c_{i_{n}} t^{i_{n}}$ directly into the modified formula (2.15) for $\mathscr{M} \Phi_{a}(s)$ instead of the defining formula (2.12), we would have found an asymptotic expansion of the same form as (3.14), but now valid further to the left. Since the two expansions coincide in their common region $\operatorname{Re} s>i_{0}$, they have to be identical. Hence we have extended the domain of validity of the expansion (3.14) to reach the next singularity of $\eta(s, a)$ left of $\left(-i_{0}\right)$; we may then repeat the argument indefinitely. Q.E.D.

\section{Zeta Functions and Functional Determinants}

We shall establish the connection of $\mathrm{Z}(s, a)$ to the canonical product determinant $\Delta(\lambda)$ in two stages. We shall first justify the definition of the functional determinant $\mathrm{D}(\lambda)$ by the zeta regularization formula [1-3], where ' denotes $d / d s$,

$$
\mathrm{D}(\lambda)=\exp \left[-\mathrm{Z}^{\prime}(0,-\lambda)\right]
$$

then we shall find that it only differs by a computable, non-constant factor from the original determinant $\Delta(\lambda)$ given by the canonical Weierstrass product.

Formally indeed, $Z^{\prime}(0,-\lambda)=-\sum_{k} \log \left(\lambda_{k}-\lambda\right)$, hence $\mathrm{D}(\lambda)=\prod_{k}\left(\lambda_{k}-\lambda\right)$ looks like a characteristic polynomial of infinite degree; but both formulae are divergent. However, Eq. (4.1) defines a finite regularization of the product, since $s=0$ is a point where the function $\mathrm{Z}(s, a)$ is analytic (thanks to the cancellation of the poles of $\eta(s, a)$ and $\Gamma(s)$, as we saw in Eq. (3.12)).

We now verify that $\mathrm{D}(\lambda)$ is indeed a determinant, more precisely that the ratio 
$\mathrm{D}(\lambda) / \Delta(\lambda)$ is a nonvanishing elementary function. We have seen previously in Eq. (2.6) that

$$
-\log \Delta(\lambda)=\left[\int_{0}^{\lambda}\right]^{[\mu]+1} R^{([\mu])}(\lambda),
$$

the $[\mu+1]$-st primitive of the convergent expression (2.5).

But $\mathrm{R}^{([\mu])}(\lambda)=\eta([\mu]+1,-\lambda)$, and we may use the functional relation (3.13) in the form

$$
\int \eta(s+1,-\lambda) d \lambda=\eta(s,-\lambda)
$$

to evaluate the successive primitives of $\eta(s,-\lambda)$, provided $s$ does not encounter poles of $\eta(s,-\lambda)$ in the process. We can thus integrate $\mathrm{R}^{[\mu]}$ at most $\mu$ times, using the boundary conditions (2.4) at $\lambda=0$, to get

$$
\begin{aligned}
\mathrm{R}_{0}(\lambda)=\left[\int_{0}^{\lambda}\right]^{[\mu]} \eta([\mu]+ & 1,-\lambda)=\eta(1,-\lambda)-\sum_{m=0}^{[\mu]-1}\left(\frac{d^{m}}{d \lambda^{m}} \mid \widehat{\lambda=0} \eta(1,-\lambda)\right) \frac{\lambda^{m}}{m !} \\
\mathrm{R}_{0}(\lambda) & =\eta(1,-\lambda)-\sum_{m=0}^{[\mu-1]} \eta(1+m, 0) \lambda^{m} / m ! \\
& =\mathrm{Z}(1,-\lambda)-\sum_{m=0}^{[\mu-1]} \mathrm{Z}(1+m) \lambda^{m}
\end{aligned}
$$

(we have used the functional relation (3.13) again).

However, we cannot integrate once more to attain $-\log \Delta(\lambda)$ because this step always produces singular quantities like $\eta(0,-\lambda)=\left.\Gamma(s) Z(s,-\lambda)\right|_{s=0}$. We then have to understand at first how to use Eq. (4.3) when poles are encountered. The simplest extension uses derivatives of continuous order [9]. We extend the definitions (2.5) and $(3.10)$ as

$$
\mathrm{R}^{(s)}(\lambda)=\Gamma(1+s) \sum\left(\lambda_{k}-\lambda\right)^{-s-1}=\eta(s+1,-\lambda) \quad(s>\mu-1) .
$$

Then $\mathrm{R}_{0}(\lambda)=\lim _{\varepsilon \rightarrow 0} \mathrm{R}_{\varepsilon}(\lambda)$, with

$$
\mathrm{R}_{\varepsilon}(\lambda)=\eta(1+\varepsilon,-\lambda)-\sum_{m=0}^{[\mu-1]} \eta(1+m+\varepsilon, 0) \lambda^{m} / m ! .
$$

In the limit $\varepsilon \rightarrow 0$, the pole terms have to cancel out in the right-hand side, hence we are left with

$$
\mathrm{R}_{0}(\lambda)=F P \eta(1,-\lambda)-\sum_{m=0}^{[\mu-1]} F P \eta(1+m, 0) \lambda^{m} / m !,
$$

where the finite part prescription is applied as usual,

$$
\left.\begin{array}{ll}
F P f(s)=f(s), & \text { if } s \text { is not a pole } \\
F P f(s)=\lim _{\varepsilon \rightarrow 0} f(s+\varepsilon)-(\text { Residue } / \varepsilon), & \text { if } s \text { is a simple pole. }
\end{array}\right\}
$$

Thus, the prescription for any formula like (4.4) is to replace $\eta(s, a)$ by its finite part whenever it has a simple pole. Caution: taking finite parts directly upon $Z(s, a)$ in formulae like (4.5) can produce different (hence wrong) results. 
Now we can perform the last integration step upon (4.7), to get

$$
-\log \Delta(\lambda)=F P \eta(0,-\lambda)-\sum_{m=0}^{[\mu]} F P \eta(m, 0) \lambda^{m} / m !
$$

There remains to express this result in terms of $Z(s, a)$ in analogy with Eq. (4.5), since we seek a relation between $\Delta$ and $Z^{\prime}$. We use $\eta(m+\varepsilon,-\lambda)=$ $\Gamma(m+\varepsilon) Z(m+\varepsilon,-\lambda)$ to compute the finite parts.

At $s=0, \Gamma(s)$ is singular and $Z(s)$ is regular,

$$
\eta(\varepsilon,-\lambda)=\left[\frac{1}{\varepsilon}-\gamma+0(\varepsilon)\right]\left[\mathrm{Z}(0,-\lambda)+\varepsilon \mathrm{Z}^{\prime}(0,-\lambda)+0\left(\varepsilon^{2}\right)\right]
$$

( $\gamma$ is the Euler constant), hence

$$
F P \eta(0,-\lambda)=Z^{\prime}(0,-\lambda)-\gamma Z(0,-\lambda)
$$

At $s=m \neq 0, \Gamma(s)$ is regular but $\mathrm{Z}(s)$ may have a pole according to (3.2),

$$
\eta(m+\varepsilon, 0)=\left[\Gamma(m)+\varepsilon \Gamma^{\prime}(m)+0\left(\varepsilon^{2}\right)\right]\left[\frac{\left(c_{-m} / \Gamma(m)\right)}{\varepsilon}+F P Z(m)+0(\varepsilon)\right],
$$

hence

$$
F P \eta(m, 0)=\Gamma(m) F P Z(m)+c_{-m} \psi(m),
$$

with $\psi=\Gamma^{\prime} / \Gamma$.

Equation (4.9) thus becomes a formula in which there finally appears the logarithm of the functional determinant $D(\lambda)$ defined by (4.1),

$$
\begin{aligned}
-\log \Delta(\hat{\lambda}) & =\mathrm{Z}^{\prime}(0,-\lambda)-\mathrm{Z}^{\prime}(0)-\sum_{m=1}^{[\mu]} F P Z(m) \lambda^{m} / m \\
& -\gamma(\mathrm{Z}(0,-\lambda)-\mathrm{Z}(0))-\sum_{m=1}^{[\mu]} c_{-m} \psi(m) \lambda^{m} / m !
\end{aligned}
$$

The second line can be still simplified, using $\mathrm{Z}(0,-\lambda)=\sum_{m=0}^{[\mu]} c_{-m} \lambda^{m} / m$ ! (Eq. (3.12)) and $\psi(m)=-\gamma+\sum_{r=1}^{m-1} r^{-1}$; we write our final result in exponentiated form,

$$
\mathrm{D}(\lambda)=e^{-\mathrm{Z}^{\prime}(0)-\sum_{m=1}^{[\mu]} F P Z(m) \lambda^{m} / m} e^{-\sum_{m=2}^{[\mu]} c_{-m}\left(1+\cdots+\frac{1}{m-1}\right) \lambda^{m} / m !} \Delta(\lambda) .
$$

We have thus expressed the ratio $\mathrm{D} / \Delta$ as the exponential of a polynomial of degree $[\mu]$ with known coefficients.

Remark. If we had blindly integrated Eq. (4.5) and used the finite part prescription upon $\mathrm{Z}(s,-\lambda)$, we would have obtained only the first exponential prefactor. However this error is quite subtle as it can only be detected with sequences of order two at least. 


\section{Semiclassical Properties of the Functional Determinant $D(\lambda)$}

In this section we show that it is the determinant $D(\lambda)$ defined by zeta regularization which has the most natural asymptotic properties for $\lambda \rightarrow-\infty$. As a consequence of the previous formula for $\mathrm{D}(\lambda) / \Delta(\lambda)$, this implies an asymptotic expansion for $\Delta(\lambda)$ itself, a kind of generalized Stirling formula.

The asymptotic expansion for $-\log \mathrm{D}(\lambda)=\mathrm{Z}^{\prime}(0,-\lambda)$ is found by differentiating the expansion (3.15) for $\mathrm{Z}(s, a)$ at $s=0$,

$$
-\log \mathrm{D}(\lambda) \underset{\lambda \rightarrow-\infty}{\sim} \sum_{i_{n} \neq-m} c_{i_{n}} \Gamma\left(i_{n}\right)(-\lambda)^{-i_{n}}-\sum_{m=0}^{[\mu]} c_{-m}\left[\log (-\lambda)-\sum_{r=1}^{m} r^{-1}\right] \frac{\lambda^{m}}{m !} ;
$$

the first sum involves all exponents $i_{n}$ except negative integers or zero, these constituting the second sum (with the convention (1.4) used throughout). We now analyze the structure of Eq. (5.1) in more detail.

We recall that $(-\log \mathrm{D})$ and $(-\log \Delta)$ have the same $[\mu+1]$-st derivative,

$$
(-\log \mathrm{D}(\lambda))^{([\mu+1])}=(-\log \Delta(\lambda))^{([\mu+1])}=\eta([\mu]+1,-\lambda) .
$$

This in turn admits the asymptotic expansion, drawn from Eq. (3.14),

$$
\mathrm{R}^{[\mu]}(\lambda)=\eta([\mu]+1,-\lambda) \sim \sum_{n=0}^{\infty} c_{i_{n}} \Gamma\left(i_{n}+[\mu]+1\right)(-\lambda)^{-i_{n}-[\mu]-1} .
$$

We now exhibit a standard integration procedure which, applied $[\mu+1]$ times to the asymptotic expansion (5.3), produces precisely the asymptotic expansion (5.1) for $-\log \mathrm{D}(\lambda)$. The idea is to choose $\lambda=-\infty$ as the lower integration bound, which is indeed the only place where those asymptotic expansions are relevant. We have for instance

$$
\int_{-\infty}^{\lambda}\left(-\lambda^{\prime}\right)^{-s} d \lambda^{\prime}=\frac{(-\lambda)^{1-s}}{s-1}
$$

but only for $s>1$. We then define the operator $\int_{-\infty}^{\lambda}$ in general as given by the analytical continuation of Eq. (5.4) in s, supplemented by finite part extractions whenever necessary. We thus obtain the set of rules

$$
\begin{gathered}
\int_{-\infty}^{\lambda} 0 d \lambda^{\prime}=0, \quad \int_{-\infty}^{\lambda}\left(-\lambda^{\prime}\right)^{-s} d \lambda^{\prime}=\frac{(-\lambda)^{1-s}}{s-1}(s \neq 1) \\
\int_{-\infty}^{\lambda}\left(-\lambda^{\prime}\right)^{-1} d \lambda^{\prime}=\underset{s \rightarrow 1}{F P}\left[\frac{(-\lambda)^{1-s}}{s-1}\right]=-\log (-\lambda) \\
\int_{-\infty}^{\lambda} \log \left(-\lambda^{\prime}\right) d \lambda^{\prime}=-\int_{-\infty}^{\lambda} \int_{-\infty}^{\lambda}\left(-\lambda^{\prime}\right)^{-1} d \lambda^{\prime}=\underset{s \rightarrow 1}{F P}\left[\frac{(-\lambda)^{2-s}}{(1-s)(2-s)}\right]=\lambda(\log (-\lambda)-1),
\end{gathered}
$$

and likewise

$$
\left(\int_{-\infty}^{\lambda}\right)^{m} \log \left(-\lambda^{\prime}\right) d \lambda^{\prime}=\frac{\lambda^{m}}{m !}\left[\log (-\lambda)-\left(1+\cdots+\frac{1}{m}\right)\right] .
$$


The so defined symbolic integration operator $\int_{-\infty}^{\lambda}$ assigns a well specified primitive to any function $f(\lambda)$ such that the divergent part of $\int_{\Lambda}^{\lambda} f\left(\lambda^{\prime}\right) d \lambda^{\prime}$ as $\Lambda \rightarrow-\infty$ has an expansion in terms of the quantities listed in Eqs. (5.5) to (5.8). (Of course, $\int_{-\infty}^{\lambda}$ has the usual meaning if the integral converges.) Similar integration procedures are well known for functions singular at a finite point [9].

Now we observe that, in order to reach $-\log \mathrm{D}(\lambda)$ by successive quadratures, we inverted the functional relation (3.13) but were unable to specify the integration constant in the resulting formula (4.3). With out new notation this ambiguity is automatically removed; indeed,

$$
\eta(s,-\lambda)=\int_{-\infty}^{\lambda} \eta\left(s+1,-\lambda^{\prime}\right) d \lambda^{\prime}
$$

because this relation is strictly true for $s>\mu$, hence it extends by analytical continuation elsewhere.

It is therefore not surprising to find, and to confirm by inspection, that

$$
-\log \mathrm{D}(\lambda)=\left(\int_{-\infty}^{\lambda}\right)^{[\mu+1]} \mathrm{R}^{([\mu])}(\lambda),
$$

to be compared with Eq. (4.2),

$$
-\log \Delta(\lambda)=\left(\int_{0}^{\lambda}\right)^{[\mu+1]} \mathrm{R}^{([\mu])}(\lambda) .
$$

We stress that Eq. (5.10) gives an effective way to compute $-\log \mathrm{D}(\lambda)$. One simply extracts from $\mathrm{R}^{[\mu]}(\lambda)$ enough leading expansion terms from Eq. (5.3) so that the remainder gives a convergent contribution to the integral (5.10), then one adds the contributions from the leading terms computed according to the special rules (5.5-5.8).

Equation (5.10) completely determines the expansion for $\mathrm{D}(\lambda)$ at $\lambda=-\infty$ in terms of the expansion for $\mathrm{R}^{([\mu])}(\lambda)$. The latter in turn has a purely semiclassical character if $\left\{\lambda_{k}\right\}$ are quantum-mechanical eigenvalues; then indeed, the coefficients $c_{i_{n}}$ are derivable by asymptotic expansion methods which become exact when $\lambda \rightarrow-\infty$ (or $t \rightarrow 0$ in the case of the partition function $\Theta(t)$ ). In fact, the numbers $c_{i_{n}}$ can often be computed in closed form $[5,6,10]$.

Thus, Eq. (5.10) means that $\log \mathrm{D}(\lambda)$ is normalized at $\lambda=-\infty$ through a standard specification for its semi-classical expansion coefficients. By contrast, $\log \Delta(\lambda)$ is normalized at the finite point $\lambda=0$ by Eq. (4.2); the difference $(\log \mathrm{D}(\lambda)-\log \Delta(\lambda))$, a polynomial of degree $[\mu]$, has coefficients (integration constants) which involve the values $\mathrm{Z}(1+m)$ as in Eq. (4.12). These constants cannot have a semiclassical character as their computation would involve integrations over a wide range of values of $\lambda$ (typically $\int_{0}^{-\infty} d \lambda$ ); in all solvable examples, those numbers $\mathrm{Z}(1+m)$ tend to be highly transcendental indeed. Consequently, the large $\lambda$ 
expansion of $\log \Delta(\lambda)$ results by combining the purely semiclassical expansion (5.1) and the exact but transcendental identity (4.12).

For all those reasons, and also because $\lambda=-\infty$ is the only translation-invariant normalization point, we should consider $\mathrm{D}(\lambda)$ as a more fundamental spectral function than $\Delta(\lambda)$. Note that under a global shift of the sequence $\left\{\lambda_{k}\right\} \rightarrow\left\{\lambda_{k}+a\right\}$, the determinant $\mathrm{D}(\lambda)$ becomes, by its definition (4.1),

$$
\mathrm{D}_{a}(\lambda)=\mathrm{D}(\lambda-a)
$$

a much simpler result than the analogous transformation rule (2.8) for $\Delta(\lambda)$.

Remark. In the light of Eq. (5.10), formula (4.12) can also be viewed as resulting from an appropriate limiting procedure $a \rightarrow+\infty$, applied to Eq. (2.8) which expresses a finite shift of the normalization point for the determinants.

We end this section by a important word of caution. When the integration operator $\int_{-\infty}^{\lambda} d \lambda^{\prime}$ is used in its symbolic extension, it does not transform as a usual integral under a change of variable; "anomalous" terms arise whenever finite parts are extracted, and indeed the canonical forms of the logarithmic terms (5.6), (5.8), are not invariant. Those correction terms are absent only for a translation of the variable, $\lambda^{\prime} \rightarrow \lambda^{\prime}+a$.

\section{Some Examples}

The output of our formalism consists essentially of two general formulae, Eq. (4.12) which relates the two determinants $\Delta(\lambda)$ and $D(\lambda)$, and Eq. (5.1) which gives the semiclassical (i.e. $\lambda \rightarrow-\infty$ ) expansion of $\mathrm{D}(\lambda)$, and thereby of $\Delta(\lambda)$.

We shall apply those formulae to a variety of examples. All the spectral sequences to be considered below have order $\mu \leqq 1$, hence our more restrictive formulae from $[6,7]$ might suffice. Still, we shall later use one basic example with order $\mu=2$, corresponding to an exotic special function known as the Barnes G-function, or double-gamma function [12,13]; however, the details of this case have been separated to form the Appendix.

The cases with $\mu<1$ are simpler since the infinite product $\Delta(\lambda)=\prod\left(1-\lambda / \lambda_{k}\right)$ and the series $\mathrm{R}(\lambda)=\sum\left(\lambda_{k}-\lambda\right)^{-1}$ both converge without need for regularization. A trivial example of this class is the spectrum of the operator $\left(-d^{2} / d \theta^{2}\right)$ on the unit circle; the eigenvalue sequence is then $\left\{\lambda_{k}=k^{2}\right\}$ and is of order $\mu=1 / 2$. A less trivial example $[6,7]$ is the spectrum of the one-dimensional Schrödinger operator $\left(-d^{2} / d x^{2}+x^{2 M}\right)(M=2,3,4 \ldots)$ which has order $\mu=(M+1) / 2 M$.

There are many interesting examples with $\mu=1$. An important case is the sequence of integers, $\left\{\lambda_{k}=k\right\}$; the spectral functions are then the basic special functions $\Gamma(a), \zeta(s) \ldots$; our treatment reproduces standard techniques and results of (elementary) analytic number theory. More interesting for us is the spectrum of the Laplace-Beltrami operator on a compact surface. Precisely, we take a surface with a Riemannian metric, denote generically by $L$ the corresponding Laplacian and focus on the spectrum of the positive operator $(-L)$. In principle our formulae applies in full generality (to operators of degree $m$ in any dimension $d$, adjusting the order as $\mu=d / m$, and to cases with boundary), but any actual computation is 
very lengthy (see for instance [5]). We prefer to treat in detail simple but far reaching special cases. Our first case will be the ordinary two-dimensional sphere; we shall connect this problem with the Barnes G-function and give the value of the related Glaisher constant in the process (see Appendix).

The other important case of interest to us, the case of compact surfaces of constant negative curvature, will be treated separately in the following section; there the formalism generates a transformation of the Selberg trace formula, which could be interesting for its own sake.

a) The Case $\left\{\lambda_{k}=k^{2}\right\}$. As explained initially, we must delete the zero mode, hence we start the indexing at $k=1$; the order of the sequence is $\mu=1 / 2$.

The partition function is related to a Jacobi theta function,

$$
\Theta(t)=\sum_{1}^{\infty} e^{-t k^{2}}=\left(\Theta_{3}\left(0 \mid \frac{i t}{\pi}\right)-1\right) / 2 .
$$

Jacobi's identity

$$
\Theta_{3}(z \mid \tau)=(-i \tau)^{-1 / 2} e^{z^{2}} / i \pi \tau \Theta_{3}\left(\frac{z}{\tau} \mid-\frac{1}{\tau}\right)
$$

([13], §21.51) then implies

$$
\Theta(t)=c_{-1 / 2} t^{-1 / 2}+c_{0}+0\left(e^{-\pi^{2} / t}\right), \quad t \rightarrow+0
$$

with the only nonvanishing expansion coefficients $c_{-1 / 2}=\sqrt{\pi} / 2, c_{0}=-\frac{1}{2}$.

We can also readily identify

$$
\Delta(\lambda)=\prod_{k=1}^{\infty}\left(1-\frac{\lambda}{k^{2}}\right)=\frac{\sin (\pi \sqrt{\lambda})}{\pi \sqrt{\lambda}} .
$$

We note that this determinant can be written as a product of two "simpler" functions. Set $\lambda=-\kappa^{2}$, and note that $\left(1+\kappa^{2} / k^{2}\right)=(1-i \kappa / k) e^{i \kappa / k}(1+i \kappa / k) e^{-i \kappa / k}$; then use the Weierstrass product formula for the gamma function as in Example c) below, to obtain

$$
\Delta(\lambda)=\frac{1}{\Gamma(1-i \kappa)} \cdot \frac{1}{\Gamma(1+i \kappa)} .
$$

This factorization thus amounts to the reflection formula for $\Gamma(z)$.

Finally,

$$
\mathrm{Z}(s)=\sum_{k=1}^{\infty} k^{-2 s}=\zeta(2 s)
$$

where $\zeta(s)$ is the Riemann zeta function.

b) The Spectrum of Homogeneous Schrödinger Operators. We consider the onedimensional Schrödinger equation

$$
-\frac{d^{2} \psi}{d x^{2}}+x^{2 M} \psi=\lambda \psi \quad(M \text { integer } \geqq 2) ;
$$

the functions $\Theta, \Delta, \mathrm{Z} \ldots$ associated to its eigenvalue sequence $\left\{\lambda_{k}\right\}$, which has order 
$\mu=(M+1) / 2 M$, do not reduce to known functions. The determinant $\mathrm{D}(\lambda)$, normalized in [6] with respect to the transmission coefficient of a certain scattering problem, does coincide with the determinant $\mathrm{D}(\lambda)$ used in this work. Our formalism produces the specific main results

$$
\begin{aligned}
& \Theta(t)=\sum_{n=0}^{\infty} c_{i_{n}} t^{i_{n}} \quad \text { with } \quad i_{n}=(2 n-1) \mu, \\
& \Delta(\lambda)=\prod_{0}^{\infty}\left(1-\lambda / \lambda_{k}\right), \quad \mathrm{D}(\lambda)=e^{-Z^{\prime}(0)} \Delta(\lambda) .
\end{aligned}
$$

However, more explicit results can be obtained for those functions by entirely different techniques [7]. For instance, $Z(1), Z(2), Z^{\prime}(0)(=\log \sin (\pi /(2 M+2)))$ are explicitly computable; the determinant $\mathrm{D}(\lambda)$ satisfies a functional equation leading to remarkable identities between the values $\mathrm{Z}(n), n=1,2, \ldots$; many asymptotic expansions can be computed, such as those for $Z(s)(s \rightarrow-\infty), c_{i_{n}}(n \rightarrow+\infty), \ldots$.

c) The Sequence of Integers $\left\{\lambda_{k}=k\right\}$. Again we index $k$ from 1 to delete the zero mode; this sequence has order $\mu=1$. A related sequence is the spectrum of the quantum harmonic oscillator, Eq. (6.6) with $M=1:\left\{\lambda_{k}=2 k+1\right\}(k=0,1, \ldots)$, which we discard here (cf. $[6,7])$.

In this case all spectral functions are classical special functions:

$$
\begin{aligned}
\Theta(t) & =\sum_{k=1}^{\infty} e^{-t k}=\frac{1}{e^{t}-1}=\sum_{n=-1}^{\infty} c_{n} t^{n}, \quad c_{n}=\frac{B_{n+1}}{(n+1) !} ; \\
\Delta(\lambda) & =\sum_{k=1}^{\infty}\left(1-\frac{\lambda}{k}\right) e^{\lambda / k}=\frac{e^{\gamma \lambda}}{\Gamma(1-\lambda)} ; \\
Z(s, a) & =\sum_{k=1}^{\infty}(k+a)^{-s}=\zeta(s, a) ; \quad(\text { and } \mathrm{Z}(s)=\zeta(s)), \\
Z(N, a) & =(-1)^{N}(N-1) ! \psi^{(N-1)}(1+a) \quad(N=2,3 \ldots) ;
\end{aligned}
$$

with standard notations [14]. The function $\mathrm{R}_{0}(\lambda)$ corresponds to the case $N=1$, where a regularization is needed according to Eq. (4.7),

$$
\mathrm{R}_{0}(\lambda)=-\psi(1-\lambda)-\gamma
$$

As for the functional determinant $\mathrm{D}(\lambda)$, Eq. (4.12) yields

$$
\mathrm{D}(\lambda)=\exp \left[-\zeta^{\prime}(0)-F P \zeta(1) \lambda\right] \Delta(\lambda) .
$$

Given that $F P \zeta(1)=\gamma$ and $\zeta^{\prime}(0)=-\frac{1}{2} \log 2 \pi$, we deduce from Eq. (6.10) that

$$
\mathrm{D}(\lambda)=\sqrt{2 \pi} / \Gamma(1-\lambda) \text {. }
$$

Taking the coefficients $c_{n}$ from Eq. (6.9), we finally recognize the asymptotic expansion (5.1) for $\mathrm{D}(-a)(a \rightarrow+\infty)$ as the Stirling formula,

$$
\log \frac{\Gamma(1+a)}{\sqrt{2 \pi}} \sim a(\log a-1)+\frac{1}{2} \log a+\sum_{n=1}^{\infty} \frac{B_{n+1}}{n(n+1)} a^{-n} .
$$

We have thus recovered classical results as special cases of a general theory. 
d) The Laplacian on the Two-Dimensional Sphere. It is well known from the theory of spherical harmonics that the spectrum of the operator $(-L)$ on the sphere is the sequence (of order $\mu=1$ ),

$$
\{l(l+1) \text { with multiplicity }(2 l+1)\} \quad l=0,1,2 \ldots .
$$

The only spectral function which is readily identifiable is

$$
Z\left(s, \frac{1}{4}\right)=\sum_{l=0}^{\infty}(2 l+1) \cdot \frac{1}{[l(l+1)+1 / 4]^{s}}=\left(2^{2 s}-2\right) \zeta(2 s-1) .
$$

This suggests taking as fundamental sequence the spectrum shifted by $+\frac{1}{4}$,

$$
\left\{\lambda_{k}\right\}=\left\{(l+1 / 2)^{2} \quad \text { with multiplicity }(2 l+1)\right\} .
$$

(it is then not necessary to remove the zero mode $l=0$ ). The previous spectral function becomes the standard zeta function for this new sequence

$$
Z(s)=\left(2^{2 s}-2\right) \zeta(2 s-1)
$$

and it has a unique pole at $s=1$ with residue 1 .

The determinant $\Delta(\lambda)$ is then given by the canonical product

$$
\Delta(\lambda)=\prod_{l=0}^{\infty}\left[\left(1-\frac{\lambda}{(l+1 / 2)^{2}}\right) e^{\lambda /(l+1 / 2)^{2}}\right]^{2 l+1} .
$$

Our main results are again the relation (4.12) of $\Delta(\lambda)$ to the other determinant $\mathrm{D}(\lambda)$, and a standard semiclassical expansion at $\lambda=-\infty$ for $\mathrm{D}(\lambda)$. In this case we know $Z(s)$ from Eq. (6.20);

$$
\begin{aligned}
Z^{\prime}(0) & =2 \log 2 \zeta(-1)-2 \zeta^{\prime}(-1)=-\frac{\log 2}{6}-2 \zeta^{\prime}(-1), \\
F P Z(1) & =2 \gamma+4 \log 2,
\end{aligned}
$$

hence

$$
\mathrm{D}(\lambda)=\exp \left[-\mathrm{Z}^{\prime}(0)-F P Z(1) \lambda\right] \Delta(\lambda)=2^{1 / 6} e^{2 \zeta^{\prime}(-1)-2(\gamma+2 \log 2) \lambda} \Delta(\lambda) .
$$

(Note that by Riemann's functional equation for $\zeta(s)$ we also have

$$
\left.\zeta^{\prime}(-1)=\frac{\zeta^{\prime}(2)}{2 \pi^{2}}+\frac{1}{12}(\log (2 \pi)+\gamma) .\right)
$$

Now, to write the asymptotic expansion (5.1) for $D(\lambda)$ we need the expansion coefficients $c_{i_{n}}$ for $\Theta(t)$. Here we do not know $\Theta(t)$, but we may use a Mellin transform argument in reverse: since $\Gamma(s) \mathrm{Z}(s)=\mathscr{M} \Theta(s)$, the coefficients $c_{i_{n}}$ must be the residues at $s=-i_{n}$ of the function $\Gamma(s) \mathrm{Z}(s)=\Gamma(s)\left(2^{2 s}-2\right) \zeta(2 s-1)$; hence $\left\{i_{n}\right\}=n-1$, and

$$
c_{-1}=1, \quad c_{n-1}=\frac{(-1)^{n-1}}{(n-1) !}\left(2^{2-2 n}-2\right) \zeta(1-2 n) \quad(n=1,2, \ldots),
$$


which can also be put together as

$$
c_{n-1}=\frac{(-1)^{n}}{n !}\left(2^{1-2 n}-1\right) B_{2 n} \quad(n=0,1,2, \ldots) .
$$

The desired expansion then reads as

$$
\log \mathrm{D}(\lambda) \sim c_{-1} \lambda(\log (-\lambda)-1)+c_{0} \log (-\lambda)-\sum_{n=2}^{\infty} c_{n-1} \Gamma(n-1)(-\lambda)^{1-n} .
$$

Next, we discuss the possible factorization of $\Delta(\lambda)$ into more elementary functions, in analogy with Eq. (6.4), which decomposed $(\sin \pi \kappa / \pi \kappa)$ into a product of gamma functions. The role of the gamma function is now played by a special function whose zeros have a linearly increasing multiplicity, like the eigenvalues of the Laplacian. One such elementary function is known as $([12,13])$

$$
\frac{1}{\Gamma_{2}(1+z)}=G(1+z)=(2 \pi)^{z / 2} e^{-1 / 2\left[(1+\gamma) z^{2}+z\right]} \prod_{n=1}^{\infty}\left[(1+z / n)^{n} e^{-z+z^{2} / 2 n}\right]
$$

(respectively called the double gamma function and the Barnes $G$-function). In the Appendix we have collected the classical properties of this function and put them in perspective with the present formalism. It is clear however that Eq. (6.21) will rather factorize through a determinant for the sequence of half-integers. The simplest choice is to select the positive frequency part of the wave operator as with Eq. (6.4), i.e. the eigenvalues of $+\sqrt{-L+1 / 4}$. These form a sequence of order 2 ,

$$
\left\{l+\frac{1}{2} \text { with multiplicity }(2 l+1)\right\}, \quad l=0,1,2 \ldots,
$$

and we may consider the associated determinant $\Delta^{+}$,

$$
\Delta^{+}(z)=\prod_{l=0}^{\infty}\left[\left(1-\frac{z}{l+1 / 2}\right) \exp \left(\frac{z}{l+1 / 2}+\frac{z^{2}}{2(l+1 / 2)^{2}}\right)\right]^{2 l+1} .
$$

On the one hand, the desired factorization is immediate,

$$
\Delta(\lambda)=\Delta^{+}(i \kappa) \Delta^{+}(-i \kappa), \quad\left(\lambda=-\kappa^{2}\right) .
$$

On the other hand, $\Delta^{+}(z)$ can be further decomposed to exhibit the Barnes $G$-function, or rather the shifted Barnes determinant,

$$
\Delta_{-1 / 2}^{B}(z)=\prod_{l=1}^{\infty}\left[\left(1-\frac{z}{l-1 / 2}\right) \exp \left(\frac{z}{l-1 / 2}+\frac{z^{2}}{2(l-1 / 2)^{2}}\right)\right]^{l},
$$

where we apply the notation (2.7) to the definition (A.19). We have indeed

$$
\Delta^{+}(z)=\Delta_{-1 / 2}^{B}(z)^{2} / \prod_{l=0}^{\infty}\left[\left(1-\frac{z}{l+1 / 2}\right) \exp \left(\frac{z}{l+1 / 2}+\frac{z^{2}}{2(l+1 / 2)^{2}}\right)\right] .
$$

Now, the shift equation (2.8) applied to the determinant (6.10) with $a=-1 / 2$ and $\mu=1$ yields the classical result

$$
\prod_{l=0}^{\infty}\left(1-\frac{z}{l+1 / 2}\right) e^{z /(l+1 / 2)}=e^{(\gamma+2 \log 2) z} \Gamma(1 / 2) / \Gamma\left(\frac{1}{2}-z\right) ;
$$


and since $\sum_{l=0}^{\infty}(l+1 / 2)^{-2}=\pi^{2} / 2$, we obtain

$$
\Delta^{+}(z)=\pi^{-1 / 2} e^{-(\gamma+2 \log 2) z-\frac{\pi^{2}}{4} z^{2}} \Gamma\left(\frac{1}{2}-z\right) \Delta_{-1 / 2}^{B}(z)^{2} .
$$

We may then express $\Delta_{-1 / 2}^{B}(z)$ in terms of $\Delta^{B}(z)$ using Eq. (2.8) again, now with $\mu=2$,

$$
\Delta_{-1 / 2}^{B}(z)=\exp \left[\mathrm{R}^{B}(1 / 2) \lambda+\left(\mathrm{R}^{B}\right)^{\prime}(1 / 2) \frac{\lambda^{2}}{2}\right] \frac{\Delta^{B}(\lambda+1 / 2)}{\Delta^{B}(1 / 2)},
$$

and $\Delta^{B}$ itself in terms of $G$ using Eq. (A.19), to obtain finally

$$
\Delta^{+}(z)=\pi^{-1 / 2}(2 \pi)^{z} e^{(1+\gamma+2 \log 2) z^{2}} \Gamma\left(\frac{1}{2}-z\right) G\left(\frac{1}{2}-z\right)^{2} / G(1 / 2)^{2},
$$

where

$$
G(1 / 2)=e^{3 \zeta^{\prime}(-1) / 2} \pi^{-1 / 4} 2^{1 / 24}
$$

from Eqs. (A.8), (A.11). More will be said about $\Delta^{+}(z)$ in the next section on the Selberg trace formula.

To summarize, $\Delta(\lambda)$ admits the following factorizations with $\lambda=-\kappa^{2}$,

$$
\begin{aligned}
& \left.\Delta(\lambda)=\Delta^{+}(i \kappa) \Delta^{+}(-i \kappa) \quad \text { Eq. }(6.32)\right), \\
& \Delta(\lambda)=\frac{e^{\pi^{2} \kappa^{2} / 2}}{\cosh \pi \kappa}\left(\Delta_{-1 / 2}^{B}(i \kappa) \Delta_{-1 / 2}^{B}(-i \kappa)\right)^{2},
\end{aligned}
$$

(by substituting (6.36) into (6.32)), and finally

$$
\Delta(\lambda)=e^{-2(1+\gamma+\log 2) \kappa^{2}} \frac{1}{\cosh \pi \kappa} \frac{1}{G(1 / 2)^{4}}\left[G\left(\frac{1}{2}+i \kappa\right) G\left(\frac{1}{2}-i \kappa\right)\right]^{2} .
$$

This result directly compares with the reflection formula (6.4) for $\Gamma^{-1}(z)$, with $\sin (\pi \sqrt{\lambda}) / \pi \sqrt{\lambda}$ (the determinant on the circle) replaced by the determinant $\Delta(\lambda)$ on the sphere. However, $G(z)$ also admits another reflection formula with a more elementary expression, but which involves the ratio $G\left(\frac{1}{2}+z\right) / G\left(\frac{1}{2}-z\right)$ rather than the product; see Eqs. (A.5).

\section{The Selberg Trace Formula}

In this section we take for $\left\{\lambda_{k}\right\}$ the sequence of eigenvalues of the operator ( - Laplacian) on a compact surface of constant negative curvature ( -1$)$. By Weyl's theorem this sequence is of order $\mu=1$.

Many properties of this sequence can be explicitly computed by virtue of the Selberg trace formula, which is a remarkable summation formula relating the value of an even function $h(\rho)$ at the points $\rho_{k}=\left(\lambda_{k}-1 / 4\right)^{1 / 2}$ to the values of its Fourier transform $\hat{h}(\tau)=(2 \pi)^{-1} \int_{-\infty}^{\infty} h(\rho) e^{i \tau \rho} d \rho$ at the lengths of the periodic geodesics on the surface $[15,16]$. Specifically, if $p$ runs over all distinct primitive, oriented, periodic geodesics and if $\tau(p)$ denotes the positive length of $p$, then every even function $h(\rho)$ analytic in $|\operatorname{Im} \rho| \leqq \frac{1}{2}+\varepsilon$, and such that $|h(\rho)| \leqq 0\left(|\rho|^{-2-\delta}\right)$ for $\rho \rightarrow \pm \infty$ satisfies 
the summation formula

$$
\sum_{k=0}^{\infty} h\left(\rho_{k}\right)=(g-1) \int_{-\infty}^{\infty}\left(-\frac{d \hat{h}}{d \tau}\right) \frac{d \tau}{\sinh \tau / 2}+\sum_{\{p\}} \sum_{n=1}^{\infty} \frac{\tau(p)}{2 \sinh \frac{n \tau(p)}{2}} \hat{h}(n \tau(p)) .
$$

Here $g$ is the genus of the surface, whose area is $4 \pi(g-1)$ by the Gauss-Bonnet theorem. This particular form is slightly at variance with the usual trace formula, where the integral term is $(g-1) \int_{-\infty}^{\infty} \rho \tanh \pi \rho h(\rho) d \rho$ (the equality of the two integrals is simply Parseval's theorem applied to the two functions $(\rho h(\rho))^{\wedge}=i d \hat{h} / d \tau$ and $\left.(\tanh \pi \rho)^{\wedge}=(2 i \pi \sinh \tau / 2)^{-1}\right)$.

It must be noted that the lowest eigenvalue is $\lambda_{0}=0$ corresponding to a constant eigenfunction (and $\rho_{0}= \pm i / 2$ ); the specific computations to be carried out here are insensitive to the presence of this zero mode, however.

The most convenient starting point is to choose

$$
h_{\lambda}(\rho)=\left(\frac{1}{4}+\rho^{2}-\lambda\right)^{-2}=\left(\rho^{2}+\kappa^{2}\right)^{-2}
$$

with

$$
\lambda=1 / 4-\kappa^{2}<0, \quad \kappa>1 / 2,
$$

so that, in the notation (2.5) with italics to distinguish from previous cases,

$$
\sum_{k} h_{\lambda}\left(\rho_{k}\right)=\sum\left(\lambda_{k}-\lambda\right)^{-2}=R^{(1)}(\lambda) \text {. }
$$

With this choice of $h_{\lambda}(\rho)$, no regularization is needed as (7.4) converges, and the Selberg trace formula becomes ([17], Eq. (VII. 20))

$$
R^{(1)}(\lambda)=2(g-1)\left(\frac{1}{2 \kappa} \frac{\partial}{\partial \kappa}\right) \psi\left(\frac{1}{2}+\kappa\right)-\sum_{\{p\}} \sum_{n=1}^{\infty} \frac{\tau(p)}{2 \sinh \frac{n \tau(p)}{2}}\left(\frac{1}{2 \kappa} \frac{\partial}{\partial \kappa}\right) \frac{e^{-\kappa n \tau(p)}}{2 \kappa} .
$$

Since $\lambda=\frac{1}{4}-\kappa^{2}$, the differentiation operator $(1 / 2 \kappa)(\partial / \partial \kappa)$ coincides with $-\partial / \partial \lambda$. An equivalent regularization of the trace formula is given in [16], Eq. (7.15).

In the right-hand side of Eq. (7.5) we transform the following sums

$$
\begin{aligned}
\tau(p) \sum_{n=1}^{\infty} \frac{e^{-n \tau(p) \kappa}}{2 \sinh \frac{n \tau(p)}{2}} & =\tau(p) \sum_{n=1}^{\infty} \sum_{k=0}^{\infty} e^{-n \tau(p)(\kappa+k+1 / 2)}=\tau(p) \sum_{k=0}^{\infty} \frac{e^{-\tau(p)(\kappa+k+1 / 2)}}{1-e^{-\tau(p)(\kappa+k+1 / 2)}} \\
& =\sum_{k=0}^{\infty} \frac{\partial}{\partial \kappa} \log \left(1-e^{-\tau(p)(\kappa+k+1 / 2)}\right) .
\end{aligned}
$$

All sums involved precisely converge for $\operatorname{Re} \kappa>1 / 2$; this is a consequence of the explicit formulae expressing the exponential proliferation of periodic orbits with increasing lengths [18].

This motivates the introduction of the Selberg zeta function [15].

$$
\mathscr{Z}(s)=\prod_{\{p\}} \prod_{k=0}^{\infty}\left(1-e^{-\tau(p)(s+k)}\right) \quad(s>1) .
$$


We emphasize that this function is not the zeta function of any sequence in the sense of Eq. (1.7); in the present context it will turn out to behave like a functional determinant.

The infinite product (7.7) converges for $\operatorname{Re} s>1$. When $s \rightarrow+\infty$ it shows that $\mathscr{Z}(s)$ tends exponentially fast to 1.

With the help of the Selberg zeta function and thanks to Eq. (7.6), the trace formula (7.5) reads as

$$
R^{(1)}(\lambda)=2(g-1)\left(\frac{1}{2 \kappa} \frac{\partial}{\partial \kappa}\right) \psi\left(\frac{1}{2}+\kappa\right)+\left(\frac{1}{2 \kappa} \frac{\partial}{\partial \kappa}\right)^{2}\left[-\log \mathscr{Z}\left(\frac{1}{2}+\kappa\right)\right] .
$$

We shall now integrate this twice with respect to $\lambda$, noting that $\int d \lambda=-\int 2 \kappa d \kappa+$ const. The structure of the final result can be immediately described by a heuristic computation. The second primitive of $R^{(1)}(\lambda)$ is given by Eq. (2.6), since $\mu=1$ in the present case, $\int d \lambda \int d \lambda R^{(1)}(\lambda)=-\log \Delta(\lambda)$, where $\Delta(\lambda)$ is the determinant of the eigenvalue sequence $\left\{\lambda_{k}\right\}$. Hence we must find ultimately

$$
-\log \Delta(\lambda)=2(g-1) \int 2 \kappa d \kappa \psi\left(\frac{1}{2}+\kappa\right)-\log \mathscr{Z}\left(\frac{1}{2}+\kappa\right)+C \kappa^{2}+C^{\prime},
$$

where $C, C^{\prime}$ are the integration constants arising from the quadrature operation $\left(\int d \lambda\right)^{2}$. Equivalently,

$$
\Delta(\lambda)=e^{-C \kappa^{2}-C^{\prime}} \mathscr{Z}\left(\frac{1}{2}+\kappa\right) / \mathscr{G}(\kappa)^{2(g-1)},
$$

where $\mathscr{G}(\kappa)$ is a function having logarithmic derivative $2 \kappa \psi\left(\frac{1}{2}+\kappa\right)$. Now, $\mathscr{G}$ can be easily expressed in terms of the Barnes $G$-function studied in the Appendix, and the only serious problem is to identify the integration constants $C, C^{\prime}$; we therefore have to perform the two integration steps with more care.

The crucial remark here is that the formalism developed in Sect. 5 is particularly well suited for integrating the Selberg trace formula. In this case indeed, the optimal choice of integration bound is $\kappa=+\infty$ (or $\lambda=-\infty$ ), precisely because

$$
\int_{\kappa}^{+\infty} 2 \kappa^{\prime} d \kappa^{\prime} \int_{\kappa^{\prime}}^{+\infty} 2 \kappa^{\prime \prime} d \kappa^{\prime \prime}\left[\left(\frac{1}{2 \kappa^{\prime \prime}} \frac{\partial}{\partial \kappa^{\prime \prime}}\right)^{2}\left(-\log \mathscr{Z}\left(\frac{1}{2}+\kappa^{\prime \prime}\right)\right)\right]=-\log \mathscr{Z}\left(\frac{1}{2}+\kappa\right) ;
$$

this is due to the exponential decrease of $-\log \mathscr{Z}\left(\frac{1}{2}+\kappa\right)$ for $\kappa \rightarrow+\infty$. On the other hand we obtain, using Eq. (5.10),

$$
\int_{-\infty}^{\lambda} d \lambda^{\prime} \int_{-\infty}^{\lambda^{\prime}} d \lambda^{\prime \prime} R^{(1)}\left(\lambda^{\prime \prime}\right) d \lambda^{\prime \prime}=-\log D(\lambda)
$$

where $D(\lambda)$ denotes the zeta-regularized determinant of the eigenvalue sequence $\left\{\lambda_{k}\right\}$.

There remains to integrate the $\psi$ term twice. We first note that

$$
\int_{\kappa}^{\infty} 2 \kappa^{\prime} d \kappa^{\prime}\left(\frac{1}{2 \kappa^{\prime}} \frac{\partial}{\partial \kappa^{\prime}}\right) \psi\left(\frac{1}{2}+\kappa^{\prime}\right)=-\psi\left(\frac{1}{2}+\kappa\right)
$$

because the Stirling expansion for $\psi\left(\frac{1}{2}+\kappa\right)$ only contains terms of the standard form (5.5), (5.6) (and no constant term). 
The second integration step produces $\int_{\kappa}^{\infty} 2 \kappa^{\prime} \psi\left(\frac{1}{2}+\kappa^{\prime}\right) d \kappa^{\prime}$. To identify this primitive, we note that $2 \kappa \psi\left(\frac{1}{2}+\kappa\right)$ has poles at $\left(-\frac{1}{2}-l\right)$ with residues $-(2 l+1)$. Hence, $\exp \left(-\int_{\kappa}^{\infty} 2 \kappa^{\prime} \psi\left(\frac{1}{2}+\kappa^{\prime}\right) d \kappa^{\prime}\right)$ has exactly the same zeros and multiplicities as the function $\Delta^{+}(-\kappa) ; \Delta^{+}(z)$ was defined by Eq. (6.31) as the determinant of $\sqrt{-L+1 / 4}, L$ being the Laplacian on the two-dimensional sphere. We then verify by a direct computation upon Eq. (6.31) that

$$
\left(\frac{1}{2 \kappa} \frac{\partial}{\partial \kappa}\right)\left(-\log \Delta^{+}(-\kappa)\right)=-\psi\left(\frac{1}{2}+\kappa\right)
$$

According to our formalism, this means that

$$
\int_{\kappa}^{\infty} 2 \kappa^{\prime} d \kappa^{\prime} \psi\left(\frac{1}{2}+\kappa^{\prime}\right)=-\log \mathrm{D}^{+}(-\kappa)
$$

where $\mathrm{D}^{+}(z)$ is the corresponding zeta-regularized determinant.

We still cannot use Eqs. (7.10), (7.11) and (7.14) alone to express the second primitive of Eq. (7.8), for the reason mentioned at the end of Sect. 5; precisely, the integration operators $\int_{-\infty}^{\lambda} d \lambda^{\prime}$ and $\int_{\kappa}^{\infty} 2 \kappa^{\prime} d \kappa^{\prime}$ are not equal when they perform divergent integrations (the change of variable $\lambda=1 / 4-\kappa^{2}$ then does not work as usual). The simplest expression of the difference is found by integrating in the two ways the asymptotic expansion of Eq. (7.8), which is generated by the Stirling expansion for $\psi\left(\frac{1}{2}+\kappa\right)$ (the exponentially decreasing $\log \mathscr{Z}$ term does not contribute),

$R^{(1)}(\lambda) \sim(g-1) \sum_{n=0}^{\infty}\left(2^{1-2 n}-1\right) B_{2 n} \kappa^{-2 n-2}=(g-1)\left[B_{0} \kappa^{-2}-\frac{B_{2}}{2} \kappa^{-4}+O\left(\kappa^{-6}\right)\right]$.

Applying the standard integration rules $(5.5-5.8)$ with the variable $-\kappa^{2}(=\lambda-1 / 4)$, we get

$$
\left(\int_{-\infty}^{\lambda}\right)^{2} R^{(1)}=(g-1)\left[B_{0} \kappa^{2}\left(\log \left(\kappa^{2}\right)-1\right)+\frac{B_{2}}{2} \log \left(\kappa^{2}\right)+O\left(\kappa^{-2}\right)\right] .
$$

(recall that the translation by $\frac{1}{4}$ does not alter the standard integration rules). If by contrast we apply those same integration rules with the variable $\kappa$, we get

$$
\left(\int_{\kappa}^{\infty} 2 \kappa^{\prime} d \kappa^{\prime}\right)^{2} R^{(1)}=(g-1)\left[2 B_{0} \kappa^{2}\left(\log \kappa-\frac{3}{2}\right)+B_{2} \log \kappa+O\left(\kappa^{-2}\right)\right] .
$$

Hence the difference between (7.16) and (7.17) is exactly $2(g-1) \kappa^{2}$ (the remainder terms being identical). Taking this into account, we can exponentiate the twice integrated expression (7.8) to finally obtain

$$
\mathscr{Z}\left(\frac{1}{2}+\kappa\right)=\left[e^{\kappa^{2}} \mathrm{D}^{+}(-\kappa)\right]^{2(g-1)} D(\lambda), \quad\left(\lambda=\frac{1}{4}-\kappa^{2}\right) .
$$

This "Selberg determinant formula" is not just the multiplicative analogue of the usual Selberg trace formula (7.5); it also contains the implicit specification of 
the two integration constants $C, C^{\prime}$ appearing in Eq. (7.9). Equation (7.18) also illuminates better the nature of the Selberg zeta function by providing for it a clean factorization; for instance, the analytical continuation of $\mathscr{Z}(s)$ to an entire function, and the well known structure of its zeros ([16], Theorem 7.2), are obvious consequences of (7.18). (This occurrence of two determinants may appear mysterious, especially for $\mathrm{D}^{+}$, but it has a dynamical explanation as a quantum tunneling phenomenon, to be given in a future publication.)

We now try to make the factorization (7.18) more explicit by expressing $\mathscr{Z}\left(\frac{1}{2}+\kappa\right)$ in terms of $\Delta$ determinants (canonical products).

This can be more easily done upon the factor containing $\mathrm{D}^{+}$, which relates to the better known Laplacian on the sphere. The determinants $\mathrm{D}^{+}$and $\Delta^{+}$are closely related to the determinants $\mathrm{D}^{B}$ and $\Delta^{B}$ associated with the Barnes $G$-function. We can then derive the ratio $\mathrm{D}^{+} / \Delta^{+}$as in the Appendix, identifying

$$
\begin{gathered}
\Theta^{+}(t)=\sum_{l=0}^{\infty}(2 l+1) e^{-\left(l+\frac{1}{2}\right) t}=-\frac{d}{d t} \frac{1}{\sinh t / 2}, \\
c_{-2}^{+}=2, \quad c_{2 n-2}^{+}=\frac{B_{2 n}\left(1-2^{1-2 n}\right)}{n(2 n-2) !} \quad(n=1,2, \ldots), \\
\mathrm{Z}^{+}(s)=\sum_{l=0}^{\infty}(2 l+1)\left(l+\frac{1}{2}\right)^{-s}=\left(2^{s}-2\right) \zeta(s-1),
\end{gathered}
$$

to obtain finally, thanks to Eqs. (6.38) and (6.39),

$$
\begin{aligned}
\mathrm{D}^{+}(z) & =2^{1 / 12} e^{\zeta^{\prime}(-1)-(1+\gamma+2 \log 2) z^{2}} \Delta^{+}(z) \\
& =e^{-2 \zeta^{\prime}(-1)}(2 \pi)^{z} \Gamma\left(\frac{1}{2}-z\right) G\left(\frac{1}{2}-z\right)^{2},
\end{aligned}
$$

an expression involving the Barnes $G$-function studied in the Appendix.

This has two interesting consequences. First, the parity of $D(\lambda)$ with respect to $\kappa$ implies that

$$
\mathscr{Z}\left(\frac{1}{2}+\kappa\right) / \mathscr{Z}\left(\frac{1}{2}-\kappa\right)=\left[\mathrm{D}^{+}(-\kappa) / \mathrm{D}^{+}(\kappa)\right]^{2 g-2} ;
$$

but Eq. (7.23) for $D^{+}$and the reflection formula (A.5) for $G(z)$ together transform this relation into the well known functional equation for $\mathscr{Z}(s)$,

$$
\mathscr{Z}\left(\frac{1}{2}+\kappa\right) / \mathscr{Z}\left(\frac{1}{2}-\kappa\right)=\exp 4(g-1) \int_{0}^{\kappa} \pi \kappa^{\prime} \tan \left(\pi \kappa^{\prime}\right) d \kappa^{\prime} .
$$

(This ratio no longer involves the constants of integration $C, C^{\prime}$ of Eq. (7.9) either; hence Eq. (7.25) is directly derivable from the original trace formula (7.8).)

Another consequence of (7.23) is a more explicit form for Eq. (7.18), [36],

$$
\mathscr{Z}\left(\frac{1}{2}+\kappa\right)=\left[e^{\kappa^{2}-2 \zeta^{\prime}(-1)}(2 \pi)^{-\kappa} \Gamma\left(\frac{1}{2}+\kappa\right) G\left(\frac{1}{2}+\kappa\right)^{2}\right]^{2(g-1)} D(\lambda), \quad\left(\lambda=\frac{1}{4}-\kappa^{2}\right) .
$$

Incidentally, this formula exhibits a close analogy (and certain differences as well) with the factorization formula for the Riemann zeta function over its nontrivial zeros $\rho$,

$$
\zeta(s)=e^{(\log 2 \pi-1-\gamma / 2) s} \frac{1}{2(s-1) \Gamma(1+s / 2)} \prod_{\rho}\left[\left(1-\frac{s}{\rho}\right) e^{s / \rho}\right]
$$


We shall now set $\kappa=1 / 2$ to reobtain certain recent results $[1,3]$ as special cases of Eq. (7.26). We must however be careful because there is a zero mode $(\lambda=0$ is a simple eigenvalue), hence both $D(\lambda)$ and $\mathscr{Z}\left(\frac{1}{2}+\kappa\right)$ have a simple zero at $\lambda=0, \kappa=1 / 2$. We thus have to check that our formalism works in this initially excluded case.

The determinant defined by Eq. (4.2) is now the Weierstrass product

$$
\Delta(\lambda)=(-\lambda) \prod_{k=1}^{\infty}\left(1-\lambda / \lambda_{\kappa}\right) e^{\lambda / \lambda_{\kappa}}=-\lambda \Delta_{*}(\lambda)
$$

where* labels spectral functions with the zero mode deleted. Hence

$$
-\log \Delta(\lambda)=-\log (-\lambda)-\log \Delta_{*}(\lambda)
$$

but since $\log (-\lambda)$ is the standard primitive of its derivative according to the rule (5.6), we then also have

$$
D(\lambda)=-\lambda D_{*}(\lambda)
$$

for the zeta regularized determinants. The comparison of Eqs. (7.28) and (7.29) confirms that the zero mode is harmless as announced previously, since it can be factorized out explicitly. (This holds for any multiplicity.)

If we now insert (7.29) into (7.26) and differentiate at $\kappa=1 / 2$, we obtain, using $G(1)=1$,

$$
\mathscr{Z}^{\prime}(1)=e^{K(g-1)} D_{*}(0), \quad K=\frac{1}{2}-4 \zeta^{\prime}(-1)-\log 2 \pi \simeq-0.6761925 .
$$

This result agrees with Eqs. (7.23) and (7.30) of Ref. [1a]; also compare with [3] (Appendix), where $K$ is only expressed as an integral,

$$
K=\pi \int_{0}^{\infty}\left(\frac{1}{4}+r^{2}\right) \operatorname{sech}^{2} \pi r\left[-1+\log \left(\frac{1}{4}+r^{2}\right)\right] d r .
$$

We now return to our more general factorization formula (7.26), and now try to make the other factor $D(\lambda)$ more explicit in terms of canonical products (this is the factor connected with the eigenvalues on the compact Riemann surface). There we can only write, using Eqs. (7.28), (7.29) and (4.12) (with $\mu=1$ ).

$$
\frac{D(\lambda)}{\Delta(\lambda)}=\frac{D_{*}(\lambda)}{\Delta_{*}(\lambda)}=e^{-Z_{*}^{\prime}(0)-F P Z_{*}(1) \lambda},
$$

where $Z_{*}(s)$ is the zeta function with the zero mode deleted,

$$
Z_{*}(s)=\sum_{k=1}^{\infty} \lambda_{k}^{-s}
$$

We then find as our final result

$$
\mathscr{Z}\left(\frac{1}{2}+\kappa\right)=\left[e^{\kappa^{2}-2 \zeta^{\prime}(-1)}(2 \pi)^{-\kappa} \Gamma\left(\frac{1}{2}+\kappa\right) G\left(\frac{1}{2}+\kappa\right)^{2}\right]^{2(g-1)} e^{-F P Z_{*}(1) \lambda-Z_{*}^{\prime}(0)} \Delta(\lambda),
$$

which has the structure predicted by Eq. (7.9), considering that $\lambda=1 / 4-\kappa^{2}$.

In conclusion, the main results of this section are the factorization formulae (7.18), (7.26) and (7.34) for the Selberg zeta function. The last obstacle which prohibits its fully explicit factorization into a canonical Weierstrass form is our 
ignorance of the two integration constants $Z_{*}^{\prime}(0)$ and $F P Z_{*}(1)$ in Eq. (7.32). Recent advances ([19] and references therein) suggest that these might be computable at least for special Riemann surfaces.

\section{Appendix. Old and New Properties of the Barnes $G$-Function}

This function is defined by the infinite product ([12], [13] Chap. XII, Ex. 48-50)

$$
G(1+z)=(2 \pi)^{z / 2} e^{-1 / 2\left[(1+\gamma) z^{2}+z\right]} \prod_{n=1}^{\infty}\left[(1+z / n)^{n} e^{-z+z^{2} / 2 n}\right] .
$$

It is an entire function analogous to $1 / \Gamma(z)$ except that it has order 2 instead of 1 , its zeros being the negative integers $(-n)$ with multiplicity $n(n=1,2,3 \ldots)$. This definition is indeed similar to the Weierstrass product for $1 / \Gamma(z)$. Its other properties are likewise similar in their nature and proof, only more complicated. We therefore list them by their familiar names in the theory of the gamma function. - normalization:

$$
G(1)=1
$$

-functional equation:

$$
G(z+1)=\Gamma(z) G(z)
$$

- Taylor series in $|z|<1$ :

$$
\log G(1+z)=(\log 2 \pi-1) \frac{z}{2}-(1+\gamma) \frac{z^{2}}{2}+\sum_{n=3}^{\infty}(-1)^{n-1} \zeta(n-1) \frac{z^{n}}{n}
$$

- reflection formula (two equivalent forms given):

$$
\begin{aligned}
& \frac{G(1+z)}{G(1-z)}=(2 \pi)^{z} \exp -\int_{0}^{z} \pi z^{\prime} \cot \pi z^{\prime} d z^{\prime}, \\
& \frac{G\left(\frac{1}{2}+z\right)}{G\left(\frac{1}{2}-z\right)}=\frac{(2 \pi)^{z}}{\Gamma\left(\frac{1}{2}+z\right)}\left(\frac{\pi}{\cos \pi z}\right)^{1 / 2} \exp \int_{0}^{z} \pi z^{\prime} \tan \pi z^{\prime} d z^{\prime} ;
\end{aligned}
$$

- Stirling expansion for $z \rightarrow+\infty$ :

$$
\log G(1+z) \sim z^{2}\left(\frac{\log z}{2}-\frac{3}{4}\right)+\frac{z}{2} \log 2 \pi-\frac{\log z}{12}+\frac{1}{12}-\log A+O\left(\frac{1}{z}\right),
$$

where

$$
\log A=\lim _{N \rightarrow \infty} \log \left(1^{1} \cdot 2^{2} \ldots N^{N}\right)-\left(\frac{N^{2}}{2}+\frac{N}{2}+\frac{1}{12}\right) \log N+\frac{N^{2}}{4} ;
$$

( $A \simeq 1.28242713$ is called the Glaisher, or Glaisher-Kinkelin, constant [21]); -special value:

$$
G(1 / 2)=A^{-3 / 2} \pi^{-1 / 4} e^{1 / 8} 2^{1 / 24} .
$$

(Many other properties exist, like the duplication formula, which will not be used here; we simply refer to the literature [12].) 
The $G$-function has been revived lately in connection with the Selberg trace formula [22], for reasons which are clear from the main text. Surprisingly though, it is little known that the Glaisher constant $A$ (analogous to the Stirling constant $\sqrt{2 \pi}$ for the gamma function) can be evaluated in a quite elementary way; this fact is actually concealed in some literature on Toeplitz determinants which also involves the Barnes $G$-function [23,24].

It is clear from Eq. (A.7) that $\log A$ must be the finite part of the divergent sum $\left(\sum n \log n\right)$ according to some regularization, hence it must be related to $-d / d s\left(\sum n^{-s}\right)_{s=-1}=-\zeta^{\prime}(-1)$. Now, to compute $\zeta^{\prime}(s)$ around $s=-1$, we may use the Euler-Mc Laurin summation formula to obtain ([25], Eq. (13.10.8) p. 333)

$$
\zeta(s)=\lim _{N \rightarrow \infty}\left[\sum_{1}^{N} n^{-s}-\frac{N^{1-s}}{1-s}-\frac{N^{-s}}{2}+\frac{s N^{-s-1}}{12}\right](s \simeq-1) .
$$

We may differentiate under the limit sign at $s=-1$ to obtain ([23], bottom of p. 280)

$$
\zeta^{\prime}(-1)=\lim \left[-\sum_{1}^{N} n \log n+\log N\left(\frac{N^{2}}{2}+\frac{N}{2}+\frac{1}{12}\right)-\frac{N^{2}}{4}+\frac{1}{12}\right] .
$$

Comparing this with the definition of $A$, we find that

$$
\log A=-\zeta^{\prime}(-1)+1 / 12
$$

An alternative expression as a convergent series follows from the functional equation for $\zeta(s)$, which relates $\zeta^{\prime}(-1)$ to $\zeta^{\prime}(2)$ by Eq. (6.25),

$$
\log A=-\frac{\zeta^{\prime}(2)}{2 \pi^{2}}+\frac{1}{12}(\log 2 \pi+\gamma) .
$$

[Remark. A classical quadrature formula ([12]; [26] formula 6.441 (4)),

$$
\log G(1+z)=z \log \Gamma(1+z)-\int_{0}^{z} \log \Gamma\left(1+z^{\prime}\right) d z^{\prime}-\frac{z(z+1)}{2}+\frac{\log 2 \pi}{2} z,
$$

together with Eqs. (A.3) and (A.11), turn a whole set of definite integrals into closed form,

$$
\begin{aligned}
\int_{0}^{n+1 / 2} \log \Gamma(1+z) d z= & \left(n+\frac{1}{2}\right) \log \Gamma\left(n+\frac{3}{2}\right)-\sum_{m=0}^{n} \log \Gamma\left(m+\frac{1}{2}\right)-\frac{(n+1 / 2)(n+3 / 2)}{2} \\
& \left.+\left(n+\frac{5}{12}\right) \frac{\log 2}{2}+\frac{(n+1)}{2} \log \pi-3 \zeta^{\prime}(-1) / 2 .\right]
\end{aligned}
$$

We now show that many properties of the $G$-function can be immediately derived from our general formalism. We exclude from these considerations the functional equation (A.3) which has a number theoretical nature (it relates to the arithmetic progression of the integers).

Indeed, it suffices to specialize our formula to the following sequence (of order $\mu=2$ ),

$$
\left\{\lambda_{k}\right\}=\{n \text { with multiplicity } n\}, \quad n=1,2,3 \ldots
$$


We distinguish the spectral functions of this sequence by the superscript $B$. We can readily identify

$$
\Theta^{B}(t)=\sum_{n=1}^{\infty} n e^{-t n}=-\frac{d}{d t}\left(\frac{1}{e^{t}-1}\right)
$$

and

$$
\mathrm{Z}^{B}(s)=\sum_{n=1}^{\infty} n \cdot n^{-s}=\zeta(s-1)
$$

The coefficients $c_{i_{n}}$ are then

$$
c_{n-2}=\frac{(1-n) B_{n}}{n !}\left(n=0,1,2, \ldots ; \text { note that } c_{-1}=c_{1}=c_{3}=\cdots=0\right) .
$$

The $G$-function itself is related to the determinant,

$$
\Delta^{B}(\lambda)=\prod_{n=1}^{\infty}\left[\left(1-\frac{\lambda}{n}\right) e^{\lambda / n+\lambda^{2} / 2 n^{2}}\right]^{n}=(2 \pi)^{\lambda / 2} e^{1 / 2\left[(1+\gamma) \lambda^{2}-\lambda\right]} G(1-\lambda),
$$

whose logarithmic derivative evaluates as

$$
\frac{d}{d \lambda}\left(-\log \Delta^{B}(\lambda)\right)=\mathrm{R}^{B}(\lambda)=\lambda(-\gamma-\psi(1-\lambda))
$$

this is just the reciprocal of the quadrature formula (A.13).

The zeta-regularized determinant is given by Eq. (4.12),

$$
\begin{aligned}
\mathrm{D}^{B}(\lambda) & =\exp \left[-\zeta^{\prime}(-1)-\zeta(0) \lambda-\left(\frac{P F \zeta(1)}{2}+\frac{1}{2}\right) \lambda^{2}\right] \Delta_{B}(\lambda) \\
& =e^{1 / 2 \log (2 \pi) \lambda-\zeta^{\prime}(-1)} G(1-\lambda),
\end{aligned}
$$

and its asymptotic expansion for $\lambda \rightarrow-\infty$ follows from Eq. (5.1),

$$
\log \mathrm{D}^{B}(\lambda) \sim c_{-2}\left[\log (-\lambda)-\frac{3}{2}\right] \frac{\lambda^{2}}{2}+c_{0} \log (-\lambda)-\sum_{n=3}^{\infty} c_{n-2} \Gamma(n-2)(-\lambda)^{2-n} .
$$

This is equivalent to the Stirling formula (A.6) for $G(1+z)$, with A specified in accordance with (A.11).

Similarly, the Taylor series around $\lambda=0$ is equivalent to Eq. (A.4),

$$
\log \mathrm{D}^{B}(\lambda)=-\zeta^{\prime}(-1)-\zeta(0) \hat{\lambda}-\frac{(1+\gamma)}{2} \lambda^{2}-\sum_{m=3}^{\infty} \frac{\zeta(m-1)}{m} \lambda^{m} .
$$

We have thus gained a better understanding of $G(z)$ by considering it as a special determinant; in this context, though, the function $D^{B}(z)$ appears to be more fundamental.

Acknowledgements. We are grateful for discussions with N. L. Balazs, P. Cartier, V. W. Guillemin, C. Itzykson, M. L. Mehta; and especially D. Fried who communicated us some results prior to publication [20]. 


\section{Note added in proof.}

Summary. The functional determinant $D(\lambda)=\exp (-d / d s Z(s,-\lambda))_{s=0}$ is an entire function of the spectral variable $\lambda$ with the following properties:

1) It can be computed by finitely many quadratures applied to the convergent expression (5.10);

2) the integration constants are determined through the compliance of $\log D(\lambda)$ to the specified expansion (5.1) for $\lambda \rightarrow-\infty$;

3 ) an alternative determinant $\Delta(\lambda)$, specified by a Weierstrass product expansion, has a computable ratio to $D(\lambda)$, Eq. (4.12).

All in all, the zeta regularization prescription appears as the limiting case of a Weierstrass canonical expansion whose reference point is sent to infinity.

This formalism can be used to simplify the Selberg trace formula by removing two spurious differentiations, thus exhibiting the Selberg zeta function as essentially the product of two functional determinants, Eq. (7.18).

Supplementary References. After completing and submitting this work, we have learnt of additional relevant references:

1) Barnes' $G$-function: its connection with Toeplitz determinants goes back to [27]; the value (A.12) for Glaisher's constant was in fact found by Glaisher ([21], 1894 reference p. 2-3; he did not use the notation $\zeta^{\prime}(2)$, using the series $\sum n^{-2} \log n$ instead, and did not observe the connection with $\zeta^{\prime}(-1)$ ); see also [28, 29].

2) Selberg's trace formula and zeta function; the trace formula (7.5) is closest to the one used in [30]. The related two parameter zeta function $Z(s, a)$ was studied in this context by Randol [31]. The problem of the Hademard factorization of $\mathscr{Z}(s)$ was raised in Refs. [32], but the crucial computations of the two integration constants has always been omitted to our knowledge, except in two very recent Refs. $[33,34]$ which were both unpublished and unknown to us when we submitted the completed article above.

First, a factorization equivalent to Eq. (7.34) was given by J. Fischer ([33], Satz 3.4.11), where the determinant $\Delta_{-1 / 4}(\lambda)$ (in the notation of our Eq. (2.7)) is exhibited in place of $\Delta(\lambda)$ and, moreover, the corresponding constants $F P Z_{*}(1,-1 / 4)$ and $Z_{*}^{\prime}(0,-1 / 4)$ are replaced by explicit limits of sums over the eigenvalues themselves (we have studied the numerical behavior of such limiting processes in [35]).

Finally, while our article was being written, P. Sarnak [34] discovered the factorization formula in the form identical to Eq. (7.26), and extended it to the tensorial-spinorial Laplacians as well.

\section{References}

1. D'Hoker, E., Phong, D. H.: (a) Multiloop amplitudes for the bosonic Polyakov string. Nucl. Phys. B269, 205-234 (1986) (b) On determinants of Laplacians on Riemann surfaces. Commun. Math. Phys. 104, 537-545 (1986)

2. Ray, D., Singer, I. M.: Ann. Math. 98, 154-177 (1973)

Donnelly, H.: Am. J. Math. 101, 1365-1379 (1979)

3. Fried, D.: Invent. Math. 84, 523-540 (1986)

4. Widder, D.: The Laplace Transform (Chap. V), Princeton. NJ: Princeton University Press 1946

5. Duistermaat, H., Guillemin, V. W.: Invent. Math. 29, 39-79 (1975)

6. Voros, A.: in: The Riemann problem.... Chudnovsky, D., Chudnovsky, G. (eds.) Lecture Notes in Mathematics Vol. 925. Berlin, Heidelberg, New York: Springer 1982

7. Voros, A.: The return of the quartic oscillator. The complex WKB method. Ann. Inst. H. Poincaré 39A, 211-338 (1983)

(especially Sects. 4, 10 and Appendices A, C, D)

8. Hille, E.: Analytic function theory, Vol. I, Chap. 8.7 and Vol. II, Chap. 14, Blaisdell 1962-1963

9. Gelfand, I. M., Shilov, G. E.: Generalized functions Vol. 1. New York: Academic Press 1964

10. Seeley, R.: AMS Proc. Symp. Pure Math. 10, 288-307 (1966)

11. Gelfand, I. M., Levitan, B. M.: Dokl. Akad. Nauk. SSSR 88, 593-596 (1953), Dikii, L. A.: Usp. Math. Nauk 13, 111-143 (1958) (Translations AMS Series 2 18, 81-115)

12. Barnes, E. W.: Q. J. Math. 31, 264-314 (1900) 
13. Whittaker, E. T., Watson, G. N.: A course of modern analysis, Cambridge: Cambridge University Press 1965

14. Erdelyi et al.: Higher transcendental functions Vol. 1, Chap. 1 (Bateman Manuscript Project), New York: McGraw Hill 1953

15. Selberg, A.: J. Ind. Math. Soc. 20, 47-87 (1956)

16. Hejhal, D. A.: Duke Math. J. 43, 441-482 (1976)

17. Balazs, N. L., Voros, A.: Chaos on the Pseudosphere. Phys. Rep. 143, 109-240 (1986)

18. Huber, H.: Math. Anal. 138, 1-26 (1959)

19. Belavin, A., Knizhnik, V.: JETP 91, 364-390 (1986); Manin, YU.: JETP Lett. 43, 161-163 (1986)

20. Fried, D.: Invent. Math. 84, 523-540 (1986)

21. Kinkelin,: J. Reine Angew. Math. (Crelle) 57, 122-138 (1860), Glaisher, J. W. L.: Messenger of Math. 6, 71-76 (1877) and 24, 1-16 (1894)

22. Cartier, P.: Analyse numérique d'un problème de valeurs propres à haute précision (Application aux fonctions automorphes), IHES preprint (1978); Vigneras, M-F.: Astérisque 61, 235-249 (1979)

23. Widom, H.: Indian Univ. Math. J. 21, 277-283 (1971)

24. Widom, H.: Am. J. Math. 95, 333-383 (1973); McCoy, B., Wu, T. T.: The two-dimensional Ising model, Cambridge, MA; Harvard University Press 1973 (page 264 and Appendix B); Dyson, F. J.: Fredholm determinants and inverse scattering problems. Commun. Math. Phys. 47, 171-183 (1976)

25. Hardy, G. H.: Divergent Series, Clarendon Press, Oxford 1949

26. Gradshteyn, I. S., Ryzhik, I. M.: Tables of integrals, series and products (Corrected and Enlarged Edition prepared by A. Jeffrey), New York: Academic Press 1980

27. Lenard, A.: Pacific J. Math. 42, 137-145 (1972)

28. Olver, F. W. J.: Asymptotics and special functions (Chap. 8, Sects. 2.2 and 3.3). New York: Academic Press 1974

29. Vardi, I.: Determinants of Laplacians and multiple gamma functions. Stanford Math. preprint (Sept. 1986), submitted to SIAM J. Math. Anal.; Weisberger, W. I.: Normalization of the path integral measure and the coupling constants for basonic strings, Nucl. Phys. B (in press) (1987)

30. Elstrodt, J.: Jber. d. Dt. Math. Verein 83, 45-77 (1981), Eq. (10.5)

31. Randol, B.: Trans. AMS 201, 241-246 (1975)

32. Selberg, A.: Lectures 1953-1954 (unpublished; private communication of J. Elstrodt); Randol, B.: Trans. AMS 233, 241-247 (1977); Elstrodt, J., Grunewald, F., Mennicke, J.: Elementary and analytic theory of numbers. Banach center publications 17, 83-120 (1985) (Warsaw)

33. Fischer, J.: Dissertation, Univ. Münster 1985 (and "An Approach to the Selberg trace formula via the Selberg zeta function", submitted to Lecture Notes in Mathematics. Berlin, Heidelberg, New York: Springer)

34. Sarnak, P.: Determinants of Laplacians, Comm. Math. Phys. (in press) (1987)

35. Balazs, N. L., Schmit, C., Voros, A.: Spectral fluctuations and zeta functions. Saclay preprint $\mathrm{PhT} / 86-156$. J. Stat. Phys. (to appear) (M. Kac memorial issue)

36. Voros, A.: Phys. Lett. B180, 245-246 (1986)

Communicated by S-T. Yau

Received August 27, 1986; note added in proof December 1, 1986 
